\title{
Use of alkylarsonium cations as probe molecules for the study of zeolites
}

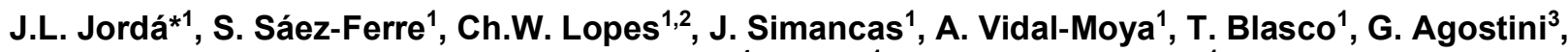 \\ G. Mínguez Espallargas ${ }^{4}, \mathrm{~F} . \mathrm{Rey}^{1}$, and P. Oña-Burgos ${ }^{1}$ \\ ${ }^{1}$ Instituto de Tecnología Química (UPV-CSIC) Universitat Politècnica de València-Consejo Superior de Investigaciones Científicas, \\ Valencia, Spain, \\ ${ }^{2}$ LRC, Institute of Chemistry, Universidade Federal do Rio Grande do Sul, Porto Alegre, Brazil, \\ ${ }^{3}$ ALBA Synchrotron Light Facility, Cerdanyola del Vallés, Barcelona, Spain, ${ }^{4}$ Instituto de Ciencia Molecular (ICMol), Universidad \\ de Valencia, Paterna, Spain \\ jjorda@itq.upv.es
}

Zeolites are crystalline microporous materials of great interest not only from an academic point of view but also from an industrial point of view due to their properties and multiple applications. These properties largely depend on its chemical composition but also on its structure. At present, the International Zeolite Association (IZA) has accepted 242 different structures [1], each with specific characteristics and a particular crystal structure. Obtaining one or the other structure is highly influenced by the organic structure directing agents (OSDAs) used during the synthesis.

Although the most typical OSDAs consist of alkylammonium cations, molecules containing phosphorous or sulfur atoms instead of nitrogen have also been described in recent years. Recently, our group also described the use of alkylarsonium cations, where nitrogen is replaced by an arsenic atom, which effectively lead to the formation of a zeolitic structure [2].

The use of As in ADE provides some additional benefits, since it allows the incorporation of heavy atoms that can act as a probe for different studies of the materials obtained. Its high electron density, compared to that of nitrogen, allows its easy location even using laboratory X-ray powder diffraction equipment; to date, the location of alkylammonium cations often requires the use of single crystal techniques or the use of complex methods. Furthermore, this substitution of $\mathrm{N}$ for As allows the use of other advanced characterization techniques, such as nuclear magnetic resonance MAS-NMR of ${ }^{75} \mathrm{As}$ in the solid sample, or X-ray absorption spectroscopy (XAS) at the $\mathrm{K}$ border of As, to analyze the location and properties of the molecule within the zeolitic network and its evolution under nonstandard conditions.

[1] http://www.iza-structure.org/databases/

[2] Sáez - Ferre S., Lopes Ch.W., Simancas J., Vidal - Moya A., Blasco T., Agostini G., Mínguez Espallargas G., Jordá JL, Rey F. and Oña - Burgos P. (2019) Use of Alkylarsonium Directing Agents for the Synthesis and Study of Zeolites. Chemistry - A European Journal 25, 16390-16396

\section{Keywords: zeolite; alkylarsonium; diffraction; XAS; NMR}

The authors acknowledge the funding of the Severo Ochoa SEV-2016-0683 and Maria de Maeztu MDM-2015-0538 programs. S.S-F thanks the MEC for its Severo Ochoa Scholarship SPV-2013-067884. P.O.-B. and G.M.E. They thank the MEC for their Ramón $y$ Cajal contracts (RYC-2014-16620 and RYC-2013-14386). The authors are grateful for the financial support of the Government of Spain (RTI2018-096399-A-I00, RTI2018-101784-B-I00 and CTQ2017-89528-P) and Generalitat Valenciana (PROMETEO / 2017/066). The UPV's Electronic Microscopy Service is thanked for its help in the characterization of samples. We thank the synchrotron ESRF for assigning the beam time (proposal CH-5193), the Italian CRG beamline in ESRF (LISA-BM08) and Alessandro Puri for help and technical support during our experiment. C.W.L. thanks CAPES for a predoctoral fellowship (Science without Frontiers - 13191/13-6). 